Ann. Funct. Anal. 6 (2015), no. 1, 249-266

http://doi.org/10.15352/afa/06-1-19

ISSN: 2008-8752 (electronic)

http://projecteuclid.org/afa

\title{
A NOTE ON FOURTH-ORDER NONLINEAR SCHRÖDINGER EQUATION
}

\author{
TAREK SAANOUNI \\ Communicated by C. Cuevas
}

\begin{abstract}
We consider in four space dimensions, the initial value problem for a fourth-order semi-linear Schrödinger equation with exponential type nonlinearity. In the defocusing sign, we obtain unconditional global well-posedness in the energy space. In the focusing case, global well-posedness via existence of ground state holds for small radial data.
\end{abstract}

\section{INTRODUCTION AND PRELIMINARIES}

1.1. Introduction. Consider the initial value problem for a semilinear fourthorder Schrödinger equation

$$
\left\{\begin{array}{c}
i \partial_{t} u+\Delta^{2} u=-\mu g(u)=:-\mu u G^{\prime}\left(|u|^{2}\right), \\
u_{0}:=u(0, .)
\end{array}\right.
$$

where $u:=u(t, x)$ is a complex-valued function of the variable $(t, x) \in \mathbb{R} \times \mathbb{R}^{4}$ and $G \in C^{2}\left(\mathbb{R}_{+}\right)$is a positive real function vanishing on zero.

Fourth-order Schrödinger equation, which is a formal extension of the classical Schrödinger equation, was introduced by Karpman[8] and Karpman and Shagalov [9] to take into account the role of small fourth-order dispersion terms in the propagation of intense laser beams in a bulk medium with Kerr nonlinearity.

Date: Received: Nov. 20, 2013; Accepted: Jan 14, 2014.

2010 Mathematics Subject Classification. Primary 35Q55; Secondary 35B30.

Key words and phrases. Fourth-order Schrödinger equation, ground state, Moser-Trudinger inequality, well-posedness. 
Any Solution to (1.1) formally satisfies the conservation of mass and energy

$$
\begin{gathered}
M(t)=M(u(t)):=\frac{1}{2}\|u(t)\|_{L^{2}\left(\mathbb{R}^{4}\right)}^{2}, \\
E(t)=E(u(t)):=\frac{1}{2}\|\Delta u(t)\|_{L^{2}\left(\mathbb{R}^{4}\right)}^{2}+\frac{\mu}{2} \int_{\mathbb{R}^{4}} G\left(|u(t)|^{2}\right) d x .
\end{gathered}
$$

If $\mu=1$, the energy is always positive and the problem (1.1) is said to be defocusing, otherwise it is focusing. In order to use the conserved quantities, we consider the usual Sobolev space $H^{2}\left(\mathbb{R}^{4}\right)$ endowed with the complete norm

$$
\|h\|_{H^{2}\left(\mathbb{R}^{4}\right)}^{2}:=\|h\|_{L^{2}\left(\mathbb{R}^{4}\right)}^{2}+\|\Delta h\|_{L^{2}\left(\mathbb{R}^{4}\right)}^{2} .
$$

Before going further let recall some historic facts about this problem.

There was an increasing activity in recent years on models involving nonlinear fourth-order partial differential equations. The book [20] by Peletier and Troy presents several such models. Fourth-order equations was also subject to an increasing activity in conformal geometry through the analysis of the Paneitz and Branson-Paneitz operators.

The model case given by a pure power nonlinearity is of particular interest. The question of well-posedness in the energy space $H^{2}$ was widely investigated. We denote for $p>1$ the fourth-order Schrödinger problem

$$
(N L S)_{p} \quad i \partial_{t} u+\Delta^{2} u \pm u|u|^{p-1}=0, \quad u: \mathbb{R} \times \mathbb{R}^{n} \rightarrow \mathbb{C} .
$$

This equation satisfies a scaling invariance. In fact, if $u$ is a solution to $(N L S)_{p}$ with data $u_{0}$, then $u_{\lambda}:=\lambda^{\frac{4}{p-1}} u\left(\lambda^{4} ., \lambda\right.$. $)$ is a solution to $(N L S)_{p}$ with data $\lambda^{\frac{4}{p-1}} u_{0}\left(\lambda\right.$.). For $s_{c}:=\frac{n}{2}-\frac{4}{p-1}$, the space $\dot{H}^{s_{c}}$ whose norm is invariant under the dilatation $u \mapsto u_{\lambda}$ is relevant in this theory. When $s_{c}=2$, which is the energy critical case, the critical power is $p_{c}:=\frac{n+4}{n-4}, n \geq 5$. Pausader [17] established global well-posedness in the defocusing subcritical case, namely $1<p<p_{c}$. Moreover, he established global well-posedness and scattering for radial data in the defocusing critical case, namely $p=p_{c}$. The same result in the critical case without radial condition was obtained by Miao, $\mathrm{Xu}$ and Zhao [14], for $n \geq 9$. The focusing case was treated by the last authors in [13]. They obtained results similar to Kenig and Merle ones [10] in the classical Schrödinger case.

Naturally, the ideas and techniques which come from the study of classical nonlinear Schrödinger equation were applied in order to study the fourth-order nonlinear Schrödinger equation.

Recall that for the monomial Schrödinger equation, local well-posedness and global well-posedness for small data were established by Cazenave and Weissler [4]. There exist a lot of works devoted to obtain global well-posedness and scattering for large data in the defocusing case [3, 6, 23, 31, 33]. However, the global well-posedness and scattering for large data in focusing case remains not completely solved. The work of Kenig and Merle [10] gives an approach of this case. When $n=2$ every polynomial nonlinearity is subcritical for classical Schrödinger 
equation. So, motivated by this fact and the so called Moser-Trudinger type inequalities [1, 16, 32], it was natural to consider nonlinearities with exponential growth.

Global well-posedness in the defocusing case was established by Nakamura and Ozawa [15] for small data, then by Colliander, Ibrahim, Majdoub and Masmoudi [5], see also [24, 27, 26, 30, 28] (similar esults hold for the wave equation $[11,12,29])$. Scattering was established in the subcritical case [7]. In the critical case a decay result was proved by the author [25]. Scattering in the critical case is obtained very recently [2].

When the space dimension is equal to four, every polynomial nonlinearity is subcritical for $(N L S)_{p}$. Motivated by this fact and the so called Moser-Trudinger type inequalities [21], we consider nonlinearities with exponential growth.

It is the goal of this paper to obtain global well-posedness in the energy space for the problem (1.1), when the nonlinearity satisfies an exponential growth. In the defocusing case unconditional well-posedness is proved. In the focusing case, global well-posedness for small radial data holds via existence of ground state. It is worth pointing out that the present study uses the potential well method based on the concepts of invariant sets suggested by Payne and Sattinger in [19].

The rest of the paper is organized as follows. The next subsection contains some notations and technical tools needed in the sequel. The second section groups the main results of this note. Section three is devoted to prove global well-posedness of (1.1) in the defocusing case. The fourth section deals with existence of a ground state solution to (1.1). In the last section we prove global well-posedness of (1.1) in the focusing case for small radial data.

We mention that $C$ (respectively $C_{T}$ ) will denote a constant (respectively a constant depending on $T$ ) which may vary from line to line and if $A$ and $B$ are nonnegative real numbers, $A \lesssim B$ means that $A \leq C B$. For $1 \leq r \leq \infty$ and $(s, T) \in[1, \infty) \times(0, \infty)$, we denote the Lebesgue space $L^{r}:=L^{r}\left(\mathbb{R}^{4}\right)$ and the usual norms

$$
\begin{gathered}
\|\cdot\|_{r}:=\|\cdot\|_{L^{r}}, \quad\|\cdot\|:=\|\cdot\|_{2}, \\
\|u\|_{L_{T}^{s}\left(L^{r}\right)}:=\left(\int_{0}^{T}\|u(t)\|_{r}^{s} d t\right)^{\frac{1}{s}}, \\
\|u\|_{L^{s}\left(L^{r}\right)}:=\left(\int_{0}^{+\infty}\|u(t)\|_{r}^{s} d t\right)^{\frac{1}{s}} .
\end{gathered}
$$

For simplicity, we denote the usual Sobolev Space $W^{s, p}:=W^{s, p}\left(\mathbb{R}^{4}\right)$ and $H^{s}:=$ $W^{s, 2}$. Note that we identify $g$ with a function on $\mathbb{R}^{2}$ and $d g$ denotes the $\mathbb{R}^{2}$ derivative of the identified function. Moreover, we denote the operator $D f(x):=$ $x f^{\prime}(x)$, where $f$ is a real function. If $X$ is an abstract space $C_{T}(X):=C([0, T], X)$ stands for the set of continuous functions valued in $X$ and $X_{r d}$ is the set of radial functions in $X$. 
1.2. Background Material. Here and hereafter, we denote for $\alpha, \beta \in \mathbb{R}$ and $v \in H^{2}$, the quantities

$$
\begin{gathered}
S(v)=\|\Delta v\|^{2}+\|v\|^{2}-\int_{\mathbb{R}^{4}} G\left(|v|^{2}\right) d x, \\
v_{\alpha, \beta}^{\lambda}:=e^{\alpha \lambda} v\left(e^{-\beta \lambda} .\right), \quad \mathcal{L}_{\alpha, \beta} S(v):=\frac{1}{2} \partial_{\lambda}\left(S\left(v_{\alpha, \beta}^{\lambda}\right)\right)_{\mid \lambda=0}, \\
K_{\alpha, \beta}(v):=\mathcal{L}_{\alpha, \beta} S(v)=\int_{\mathbb{R}^{4}}\left[\alpha|\Delta v|^{2}+(\alpha+2 \beta)|v|^{2}-\alpha|v| g(|v|)-2 \beta G\left(|v|^{2}\right)\right] d x, \\
K_{\alpha, \beta}^{Q}(v):=\int_{\mathbb{R}^{4}}\left[\alpha|\Delta v|^{2}+(\alpha+2 \beta)|v|^{2}\right] d x, \\
H_{\alpha, \beta}(v):=\left(1-\frac{\mathcal{L}_{\alpha, \beta}}{\alpha+2 \beta}\right) S(v)=\frac{1}{\alpha+2 \beta}\left[2 \beta\|\Delta v\|^{2}+\alpha \int\left(|v| g(|v|)-G\left(|v|^{2}\right)\right) d x\right], \\
m_{\alpha, \beta}^{N}:=\inf _{0 \neq v \in H_{r d}^{2}}\left\{S(v), \quad \text { s.t } \quad K_{\alpha, \beta}(v)=0\right\}, \\
A_{\alpha, \beta}^{+}:=\left\{v \in H_{\mathbb{R}^{4}}^{2} \quad \operatorname{s.} \mathrm{t} S(v)<m_{\alpha, \beta} \quad \text { and } \quad K_{\alpha, \beta}(v) \geq 0\right\} .
\end{gathered}
$$

Let fix the set of nonlinearity considered along this paper.

(1) Behavior on zero

$$
G^{\prime}(0)=G^{\prime \prime}(0)=0
$$

(2) Existence condition, $\forall \varepsilon>0, \exists C_{\varepsilon}>0$ such that

$$
\left|d^{2} g\left(z_{1}\right)-d^{2} g\left(z_{2}\right)\right| \leq C_{\varepsilon}\left|z_{1}-z_{2}\right|\left(e^{\varepsilon\left|z_{1}\right|^{2}}-1+e^{\varepsilon\left|z_{2}\right|^{2}}-1\right), \forall z_{1}, z_{2} \in \mathbb{C} .
$$

(3) Ground state condition

$$
\left\{\begin{array}{c}
\forall \alpha>0, \exists C_{\alpha}>0 /|g(s)| \leq C_{\alpha} e^{\alpha s^{2}}, \forall s \in \mathbb{R} \\
\exists \varepsilon>0 \text { s. t }(D-1-\varepsilon) G>0 \text { and }(D-1)^{2} G>0, \quad \text { on } \quad \mathbb{R}_{+}^{*} .
\end{array}\right.
$$

Remark 1.1. The function $G(r):=e^{(1+r)^{\frac{1}{2}}}-\frac{e}{2} r-e$ is an explicit example.

Proof. For $t:=\sqrt{r+1}$, we have $G(r)=e^{t}-\frac{e}{2} t^{2}-\frac{e}{2}$. Thus, $D G(r)=\frac{t^{2}-1}{2 t}\left(e^{t}-e t\right)$. Then, for $\varepsilon>0$,

$$
\begin{gathered}
\phi(t)=2(D-1-\varepsilon) G(r)=e^{t}\left(t-\frac{1}{t}-2-2 \varepsilon\right)+e\left(\varepsilon t^{2}+2+\varepsilon\right), \quad t \geq 1 \\
\phi^{\prime}(t)=e^{t}\left(t-\frac{1}{t}+\frac{1}{t^{2}}-1-2 \varepsilon\right)+2 e \varepsilon t, \\
\phi^{\prime \prime}(t)=e^{t}\left(t-\frac{1}{t}+\frac{2}{t^{2}}-\frac{2}{t^{3}}-2 \varepsilon\right)+2 e \varepsilon \geq 0 .
\end{gathered}
$$


Since $\phi(1)=\phi^{\prime}(1)=0$, we have $\phi \geq 0$. Moreover,

$$
\begin{gathered}
D(D-1) G(r)=\frac{1}{4} e^{t}\left(t-\frac{1}{t}\right)\left(t-1-\frac{1}{t}+\frac{1}{t^{2}}\right), \\
(D-1)^{2} G(r)=\frac{1}{4}\left[e^{t}\left(t^{2}-3 t+2+\frac{4}{t}+\frac{1}{t^{2}}-\frac{1}{t^{3}}\right)-4 e\right]:=\frac{1}{4} \psi(t), \\
\psi^{\prime}(t)=e^{t}\left(t^{2}-t-1+\frac{4}{t}-\frac{3}{t^{2}}-\frac{3}{t^{3}}+\frac{3}{t^{4}}\right) \geq 0 .
\end{gathered}
$$

Since $\psi(1)=0$, we have $\psi \geq 0$.

In what follows, we collect some estimates needed in the sequel.

Definition 1.2. A couple of real numbers $(q, r)$ is said to be admissible if

$$
2 \leq q, r \leq \infty,(q, r) \neq(2, \infty) \text { and } \frac{1}{q}+\frac{1}{r}=\frac{1}{2} .
$$

In order to estimate a possible solution of (1.1), we will use the following Strichartz estimates (see for example [18]).

Proposition 1.3. Let two admissible pairs $(q, r),(a, b)$ and $T>0$. There exists a positive real number $C$ such that

$$
\|u\|_{L_{T}^{q}\left(W^{2, r}\right)} \leq C\left(\left\|u_{0}\right\|_{H^{2}}+\left\|i \partial_{t} u+\Delta_{x}^{2} u\right\|_{L_{T}^{a^{\prime}\left(W^{2, b^{\prime}}\right)}}\right) .
$$

In particular, we have the following energy estimate

Proposition 1.4 .

$$
\|u\|_{L_{T}^{\infty}\left(H^{2}\right)}+\|u\|_{L_{T}^{6}\left(W^{2,3}\right)} \leq C\left(\left\|u_{0}\right\|_{H^{2}}+\left\|i \partial_{t} u+\Delta_{x}^{2} u\right\|_{L_{T}^{1}\left(H^{2}\right)}\right) .
$$

In order to control the nonlinear part of the energy in the space $L_{t}^{1}\left(H^{2}\right)$, we will use the following Moser-Trudinger inequality [21, 22, 1, 16, 32].

Proposition 1.5. For any positive real number $\alpha \leq 32 \pi^{2}$, we have

$$
\mathcal{K}:=\sup _{u \in H^{2},\|u-\Delta u\| \leq 1} \int_{\mathbb{R}^{4}}\left(e^{\alpha|u(x)|^{2}}-1\right) d x<\infty .
$$

Moreover, this is false for $\alpha>32 \pi^{2}$.

Recall some Sobolev embeddings.

Proposition 1.6. The continuous injection

$$
W^{s, p}\left(\mathbb{R}^{d}\right) \hookrightarrow L^{q}\left(\mathbb{R}^{d}\right)
$$

holds whenever

$$
1<p<q<\infty, s>0 \text { and } \quad \frac{1}{p} \leq \frac{1}{q}+\frac{s}{d} .
$$

We end this section with the generalized Pohozaev identity.

Proposition 1.7. Let $\phi \in H^{2}$ a solution to (2.1). Then, $K_{\alpha, \beta}(\phi)=0$ for any $\alpha, \beta \in \mathbb{R}$. 
Proof. Take the action

$$
S(v):=\|\Delta v\|^{2}+\|v\|^{2}-\int_{\mathbb{R}^{4}} G\left(|v|^{2}\right) d x .
$$

Then, $S^{\prime}(v) u=2 \Re\left(\left\langle\Delta^{2} v+v-g(v), u\right\rangle_{L^{2}}\right)$, so if $\phi$ is a solution to (2.1) then $S^{\prime}(\phi)=0$. Compute for $\alpha, \beta, \lambda \in \mathbb{R}$,

$$
\begin{gathered}
S\left(v_{\alpha, \beta}^{\lambda}\right)=e^{2 \alpha \lambda}\|\Delta v\|^{2}+e^{2(\alpha+2 \beta) \lambda}\|v\|^{2}-e^{4 \beta \lambda} \int_{\mathbb{R}^{4}} G\left(e^{2 \alpha \lambda}|v|^{2}\right) d x, \\
\frac{1}{2} \partial_{\lambda} S\left(v_{\alpha, \beta}^{\lambda}\right)=\alpha e^{2 \alpha \lambda}\|\Delta v\|^{2}+(\alpha+2 \beta) e^{2(\alpha+2 \beta) \lambda}\|v\|^{2} \\
-2 \beta e^{4 \beta \lambda} \int_{\mathbb{R}^{4}} G\left(e^{2 \alpha \lambda}|v|^{2}\right)-\alpha e^{2(2 \beta+\alpha) \lambda} \int_{\mathbb{R}^{4}}|v|^{2} G^{\prime}\left(e^{2 \alpha \lambda}|v|^{2}\right) d x, \\
\frac{1}{2} \partial_{\lambda} S\left(v_{\alpha, \beta}^{\lambda}\right)_{\mid \lambda=0}=\alpha\|\Delta v\|^{2}+(\alpha+2 \beta)\|v\|^{2}-\int_{\mathbb{R}^{4}}\left(2 \beta G\left(|v|^{2}\right)+\alpha|v|^{2} G^{\prime}\left(|v|^{2}\right)\right) d x .
\end{gathered}
$$

Since $\phi$ is solution to (2.1), we have $S^{\prime}(\phi)=0$. Then $\partial_{\lambda} S\left(\phi_{\alpha, \beta}^{\lambda}\right)_{\mid \lambda=0}=0$, which closes the proof.

\section{MAin RESUlts}

The results proved in this manuscript are listed in this section. Our first result is about global well-posedness of the fourth-order Schrödinger problem (1.1) in the energy space.

Theorem 2.1. Let $u_{0} \in H^{2}$ and $g$ satisfying (1.4). Then, the fourth-order Schrödinger problem (1.1) has a unique maximal solution $u$ in the energy space

$$
C\left(\left[0, T^{*}\right), H^{2}\right) \text {. }
$$

Moreover,

(1) $u$ belongs to the Strichartz space $L^{6}\left(\left(0, T^{*}\right), W^{2,3}\right)$;

(2) in the defocusing case $u$ is global $\left(T^{*}=\infty\right)$;

(3) $u$ satisfies conservation of the mass and the energy.

Next, we are interested on the focusing sign in (1.1), which is related to the stationary problem

$$
\Delta^{2} \phi+\phi=g(\phi), \quad 0 \neq \phi \in H^{2} .
$$

In fact, if $\phi$ is solution to (2.1), then $e^{-i t} \phi$ is a global solution to (1.1) called soliton or standing wave. We prove that (2.1) has a ground state in the meaning that it has a nontrivial positive radial solution which minimizes the energy with some restraint. The next result guarantees the existence of ground state.

Theorem 2.2. Let two real numbers $(0,0) \neq(\alpha, \beta) \in \mathbb{R}_{+}^{2}$. Assume that $G$ satisfies (1.5) and (1.3). Then,

(1) $m:=m_{\alpha, \beta}$ is nonzero and independent of $(\alpha, \beta)$.

(2) There is a minimizer of (1.2), which is some solution to (2.1).

The last result is about global existence of solution to the Schrödinger problem (1.1) in the focusing case. 
Theorem 2.3. Assume that $G$ satisfies (1.3)-(1.5). Let $u_{0} \in H_{r d}^{2}$ and $u \in$ $C_{T^{*}}\left(H^{2}\right)$ the maximal solution to (1.1). If there exist $(0,0) \neq(\alpha, \beta) \in \mathbb{R}_{+}^{2}$ and $t_{0} \in\left[0, T^{*}\right)$ such that $u\left(t_{0}\right) \in A_{\alpha, \beta}^{+}$, then $u$ is global.

\section{Global Well-Posedness in the Defocusing CASE}

In this section, we prove that (1.1) has a unique local solution in the energy space $C\left(\left[0, T^{*}\right), H^{2}\right)$, moreover the solution is global in the defocusing case. First, we prove the local existence by a fixed point argument.

3.1. Local existence. Recall that, for $T>0$, the space

$$
\mathcal{E}_{T}:=C\left([0, T], H^{2}\right) \cap L^{6}\left([0, T], W^{2,3}\right)
$$

is complete under the norm

$$
\|h\|_{T}:=\sup _{t \in[0, T]}\|h(t, .)\|_{H^{2}}+\|h\|_{L_{T}^{6}\left(W^{2,3}\right)} .
$$

We denote by $\mathcal{E}_{T}(1)$ the unit ball of $\mathcal{E}_{T}$ with center zero and $w$ the solution of the following free fourth-order Schrödinger problem

$$
i \partial_{t} w+\Delta^{2} w=0, \quad w(0, .)=u_{0} .
$$

We consider the map $\phi$ on $\mathcal{E}_{T}(1)$ given by $\phi(v)=\tilde{v}$, where $\tilde{v}$ solves

$$
i \partial_{t} \tilde{v}+\Delta^{2} \tilde{v}=g(v+w), \quad \tilde{v}(0, .)=0 .
$$

We prove that the map $\phi$ leaves $\mathcal{E}_{T}(1)$ stable and is a contraction for $T$ sufficiently small. Applying the energy and Strichartz estimates (1.6)-(1.7) to $v_{1}, v_{2} \in \mathcal{E}_{T}(1)$, we get

$$
\left\|\tilde{v}_{1}-\tilde{v}_{2}\right\|_{T} \lesssim\left\|g\left(v_{1}+w\right)-g\left(v_{2}+w\right)\right\|_{L_{T}^{1}\left(H^{2}\right)}:=\left\|g\left(u_{1}\right)-g\left(u_{2}\right)\right\|_{L_{T}^{1}\left(H^{2}\right)} .
$$

Using Hölder inequality via (1.4), we deduce that for any $\varepsilon>0$,

$$
\begin{aligned}
\left\|g\left(u_{1}\right)-g\left(u_{2}\right)\right\|^{2} & \lesssim C_{\varepsilon}\left\|\left|u_{1}-u_{2}\right|^{2} \sum_{i=1,2}\left(\mathrm{e}^{\varepsilon\left|u_{i}\right|^{2}}-1\right)\right\|_{1} \\
& \lesssim C_{\varepsilon} \sum_{i=1,2}\left\|\left|u_{1}-u_{2}\right|^{2}\left(\mathrm{e}^{\varepsilon\left|u_{i}\right|^{2}}-1\right)\right\|_{1} \\
& \lesssim C_{\varepsilon}\left\|u_{1}-u_{2}\right\|_{4}^{2} \sum_{i=1,2}\left\|\mathrm{e}^{2 \varepsilon\left|u_{i}\right|^{2}}-1\right\|_{1}^{\frac{1}{2}}
\end{aligned}
$$

On other hand, using the energy conservation, we get

$$
\begin{aligned}
\left\|u_{i}-\Delta u_{i}\right\|^{2} & \leq\left(\left\|u_{i}\right\|+\left\|\Delta u_{i}\right\|\right)^{2} \\
& \leq 2\left\|u_{i}\right\|_{H^{2}}^{2} \\
& \leq 2\left(1+\left\|u_{0}\right\|_{H^{2}}\right)^{2}
\end{aligned}
$$

Now, let

$$
\varepsilon_{0}:=\frac{\pi^{2}}{\left(1+\left\|u_{0}\right\|_{H^{2}\left(\mathbb{R}^{4}\right)}\right)^{2}} .
$$


Using Moser-Trudinger inequality (1.8) we have

$$
\begin{aligned}
\left\|\mathrm{e}^{2 \varepsilon_{0}\left|u_{i}\right|^{2}}-1\right\|_{1} & =\int_{\mathbb{R}^{4}}\left(\mathrm{e}^{2 \varepsilon_{0}\left\|u_{i}-\Delta u_{i}\right\|^{2}\left(\frac{\left|u_{i}\right|}{\left\|u_{i}-\Delta u_{i}\right\|}\right)^{2}}-1\right) d x \\
& \leq \mathcal{K}
\end{aligned}
$$

Thus, by (3.1),

$$
\left\|g\left(u_{1}\right)-g\left(u_{2}\right)\right\|_{L_{T}^{1}\left(L^{2}\right)} \lesssim T\left\|u_{1}-u_{2}\right\|_{T} .
$$

It remains to estimate $\left\|\Delta\left(g\left(u_{1}\right)-g\left(u_{2}\right)\right)\right\|$.

We identify $\mathbb{C}$ with $\mathbb{R}^{2}, g$ with a function on $\mathbb{R}^{2}, g^{\prime}$ with $d g$ and $g^{\prime \prime}$ with $d^{2} g$. We have

$$
\begin{aligned}
\left\|\Delta\left(g\left(u_{1}\right)-g\left(u_{2}\right)\right)\right\| & \leq\left\|\Delta\left(u_{1}-u_{2}\right) g^{\prime}\left(u_{1}\right)\right\|+\left\|\Delta u_{2}\left(g^{\prime}\left(u_{1}\right)-g^{\prime}\left(u_{2}\right)\right)\right\| \\
& +\left\|\left(\left|\nabla u_{1}\right|^{2}-\left|\nabla u_{2}\right|^{2}\right) g^{\prime \prime}\left(u_{1}\right)\right\|+\left\|\left.\nabla u_{2}\right|^{2}\left(g^{\prime \prime}\left(u_{1}\right)-g^{\prime \prime}\left(u_{2}\right)\right)\right\| \\
& \leq(I)+(I I)+(I I I)+(I V) .
\end{aligned}
$$

Now, by Moser-Trudinger inequality (1.8), via (1.4),

$$
\begin{aligned}
\|(I)\|_{L_{T}^{1}} & \leq\left\|\Delta\left(u_{1}-u_{2}\right)\left(e^{\frac{\varepsilon_{0}}{6}\left|u_{1}\right|^{2}}-1\right)\right\|_{L_{T}^{1}\left(L^{2}\right)} \\
& \leq\left\|\Delta\left(u_{1}-u_{2}\right)\right\|_{L_{T}^{1}\left(L^{3}\right)}\left\|e^{\frac{\varepsilon_{0}}{6}\left|u_{1}\right|^{2}}-1\right\|_{L_{T}^{\infty}\left(L^{6}\right)} \\
& \lesssim\left\|u_{1}-u_{2}\right\|_{T} T^{\frac{5}{6}}
\end{aligned}
$$

With the same way, via Hölder inequality

$$
\begin{aligned}
(I I) & =\left\|\Delta u_{2}\left(g^{\prime}\left(u_{1}\right)-g^{\prime}\left(u_{2}\right)\right)\right\| \\
& \leq\left\|\Delta u_{2}\right\|_{3}\left\|g^{\prime}\left(u_{1}\right)-g^{\prime}\left(u_{2}\right)\right\|_{6} \\
& \leq\left\|\Delta u_{2}\right\|_{3} \sum_{i}\left\|\left(u_{1}-u_{2}\right)\left(e^{\frac{\varepsilon_{0}}{12}\left|u_{i}\right|^{2}}-1\right)\right\|_{6} \\
& \leq\left\|\Delta u_{2}\right\|_{3}\left\|u_{1}-u_{2}\right\|_{H^{2}} \sum_{i}\left\|e^{\varepsilon_{0}\left|u_{i}\right|^{2}}-1\right\|_{1}^{\frac{1}{12}} \\
& \lesssim\left\|\Delta u_{2}\right\|_{3}\left\|u_{1}-u_{2}\right\|_{T} .
\end{aligned}
$$

Thus

$$
\begin{aligned}
\|(I I)\|_{L_{T}^{1}} & \lesssim\left\|u_{1}-u_{2}\right\|_{T}\left\|\Delta u_{2}\right\|_{L_{T}^{6}\left(L^{3}\right)} T^{\frac{5}{6}} \\
& \lesssim\left(1+\left\|u_{0}\right\|_{H^{2}}\right)\left\|u_{1}-u_{2}\right\|_{T} T^{\frac{5}{6}}
\end{aligned}
$$

Arguing as previously,

$$
\begin{aligned}
(I I I) & =\left\|\left(\left|\nabla u_{1}\right|^{2}-\left|\nabla u_{2}\right|^{2}\right) g^{\prime \prime}\left(u_{1}\right)\right\| \\
& \lesssim\left\|\left|\nabla\left(u_{1}-u_{2}\right)\right|\left(\left|\nabla u_{1}\right|+\left|\nabla u_{2}\right|\right) g^{\prime \prime}\left(u_{1}\right)\right\| \\
& \lesssim\left\|\left|\nabla\left(u_{1}-u_{2}\right)\right|\left(\left|\nabla u_{1}\right|+\left|\nabla u_{2}\right|\right)\left(e^{\frac{\varepsilon_{0}}{6}\left|u_{1}\right|^{2}}-1\right)\right\| .
\end{aligned}
$$

Using the Sobolev embedding

$$
W^{1,3}\left(\mathbb{R}^{4}\right) \hookrightarrow L^{q}\left(\mathbb{R}^{4}\right), \quad \forall 1<q \leq 12,
$$


via the interpolation inequality $\|u v w\| \leq\|u\|_{6}\|v\|_{6}\|w\|_{6}$, we have

$$
\begin{aligned}
\|(I I I)\|_{L_{T}^{1}} & \lesssim\left\|\nabla\left(u_{1}-u_{2}\right)\right\|_{L_{T}^{6}\left(L^{6}\right)}\left\|\nabla u_{1}\right\|_{L_{T}^{6}\left(L^{6}\right)}\left\|e^{\varepsilon\left|u_{1}\right|^{2}}-1\right\|_{L_{T}^{\infty}\left(L^{1}\right)}^{\frac{1}{6}} T^{\frac{2}{3}} \\
& \lesssim\left(1+\left\|u_{0}\right\|_{H^{2}}\right)\left\|u_{1}-u_{2}\right\|_{T} T^{\frac{2}{3}}
\end{aligned}
$$

By Moser-Trudinger inequality (1.8),

$$
\begin{aligned}
(I V) & =\left\|\left|\nabla u_{2}\right|^{2}\left(g^{\prime \prime}\left(u_{1}\right)-g^{\prime \prime}\left(u_{1}\right)\right)\right\| \\
& \lesssim\left\|\left(u_{1}-u_{2}\right)\left|\nabla u_{2}\right|^{2} \sum_{i}\left(e^{\frac{\varepsilon_{0}}{6}\left|u_{i}\right|^{2}}-1\right)\right\| \\
& \lesssim \sum_{i}\left\|u_{1}-u_{2}\right\|_{6}\left\|\nabla u_{2}\right\|_{12}^{2}\left\|e^{\frac{\varepsilon_{0}}{6}\left|u_{i}\right|^{2}}-1\right\|_{6} \\
& \lesssim\left\|u_{1}-u_{2}\right\|_{6}\left\|\nabla u_{2}\right\|_{12}^{2} .
\end{aligned}
$$

Thus, by the Sobolev embeddings

$$
\begin{gathered}
W^{1,3}\left(\mathbb{R}^{4}\right) \hookrightarrow L^{q}\left(\mathbb{R}^{4}\right), \quad \forall q \in(1,12], \\
H^{2}\left(\mathbb{R}^{4}\right) \hookrightarrow L^{q}\left(\mathbb{R}^{4}\right), \quad \forall q \in(2, \infty),
\end{gathered}
$$

we get

$$
\begin{aligned}
\|(I V)\|_{L_{T}^{1}} & \lesssim\left\|u_{1}-u_{2}\right\|_{L_{T}^{\infty}\left(L^{6}\right)}\left\|\nabla u_{2}\right\|_{L_{T}^{6}\left(L^{12}\right)}^{2} T^{\frac{2}{3}} \\
& \lesssim\left(1+\left\|u_{0}\right\|_{H^{2}}\right)^{2}\left\|u_{1}-u_{2}\right\|_{T} T^{\frac{2}{3}}
\end{aligned}
$$

Thus, for $T>0$ small enough, $\phi$ is a contraction satisfying

$$
\left\|\phi\left(v_{1}\right)-\phi\left(v_{2}\right)\right\|_{T} \lesssim\left(1+\left\|u_{0}\right\|_{H^{2}}\right)^{2}\left\|v_{1}-v_{2}\right\|_{T} T^{\frac{2}{3}} .
$$

Taking in the last inequality $v_{2}=0$, yields

$$
\left\|\phi\left(v_{1}\right)\right\|_{T} \lesssim\left(1+\left\|u_{0}\right\|_{H^{2}}\right)^{2} T^{\frac{2}{3}}+\|g(w)\|_{L_{T}^{1} H^{2}}
$$

Let estimate the quantity

$$
\|g(w)\|_{L_{T}^{1} H^{2}}:=\|g(w)\|_{L_{T}^{1}\left(L^{2}\right)}+\|\Delta(g(w))\|_{L_{T}^{1}\left(L^{2}\right)} .
$$

Using Moser-Trudinger inequality (1.8) via (1.4) and (3.2), we deduce that

$$
\begin{aligned}
\|g(w)\|^{2} & \lesssim C_{0} \int_{\mathbb{R}^{4}}\left(\mathrm{e}^{\varepsilon_{0}|w|^{2}}-1\right) d x \\
& \lesssim C_{0} \int_{\mathbb{R}^{4}}\left(\mathrm{e}^{\varepsilon_{0}\|w-\Delta w\|^{2}\left(\frac{|w|}{\|w-\Delta w\|}\right)^{2}}-1\right) d x \\
& \lesssim C_{0} \mathcal{K} .
\end{aligned}
$$

Where $C_{0}$ depends only on $\left\|u_{0}\right\|_{H^{2}}$. Moreover,

$$
\begin{aligned}
\|\Delta(g(w))\| & \leq\left\|\Delta w g^{\prime}(w)\right\|+\left\||\nabla w|^{2} g^{\prime \prime}(w)\right\| \\
& \leq(I)+(I I) .
\end{aligned}
$$


Now, by Moser-Trudinger inequality (1.8) via (1.4),

$$
\begin{aligned}
\|(I)\|_{L_{T}^{1}} & \leq\left\|\Delta w\left(e^{\frac{\varepsilon_{0}}{6}\left|u_{1}\right|^{2}}-1\right)\right\|_{L_{T}^{1}\left(L^{2}\right)} \\
& \leq\|\Delta w\|_{L_{T}^{1}\left(L^{3}\right)}\left\|e^{\frac{\varepsilon_{0}}{6}\left|u_{1}\right|^{2}}-1\right\|_{L_{T}^{\infty}\left(L^{6}\right)} \\
& \lesssim\|w\|_{T} T^{\frac{5}{6}} \lesssim\left\|u_{0}\right\|_{H^{2} T^{\frac{5}{6}}}
\end{aligned}
$$

With the same way, via Hölder inequality

$$
\begin{aligned}
(I I) & =\left\||\nabla w|^{2} g^{\prime \prime}(w)\right\| \\
& \lesssim\|\nabla w\|_{6}^{2}\left\|g^{\prime \prime}(w)\right\|_{6} \\
& \lesssim\|\nabla w\|_{6}^{2}\left\|e^{\frac{\varepsilon_{0}}{6}|w|^{2}}-1\right\|_{6} \lesssim\|\nabla w\|_{6}^{2} .
\end{aligned}
$$

Using the Sobolev injection

$$
W^{1,3}\left(\mathbb{R}^{4}\right) \hookrightarrow L^{q}\left(\mathbb{R}^{4}\right) \quad \text { for any } \quad q \in(1,12),
$$

yields

$$
\|(I I)\|_{L_{T}^{1}} \lesssim\|w\|_{L_{T}^{6} W^{2,3}}^{2} T^{\frac{2}{3}} \lesssim\left\|u_{0}\right\|_{H^{2}}^{2} T^{\frac{2}{3}}
$$

Finally, we have $\|g(w)\|_{L_{T}^{1} H^{2}} \lesssim\left\|u_{0}\right\|_{H^{2}}\left(1+\left\|u_{0}\right\|_{H^{2}}\right) T^{\frac{2}{3}}$. Then, for $T>0$ small enough, $\phi$ maps the unit ball into itself, in fact

$$
\left\|\phi\left(v_{1}\right)\right\|_{T} \lesssim\left(1+\left\|u_{0}\right\|_{H^{2}}\right)^{2} T^{\frac{2}{3}}+\|g(w)\|_{L_{T}^{1} H^{2}} \lesssim\left(1+\left\|u_{0}\right\|_{H^{2}}\right)^{2} T^{\frac{2}{3}}
$$

So, for small time $\phi$ is a contraction of the unit ball. Existence of solution to the fourth-order Schrödinger problem (1.1) follows by a classical Theorem of Picard.

3.2. Uniqueness in the energy space. The uniqueness proof is a consequence of the following Lemma and the proof of local existence of solution to (1.1).

Lemma 3.1. Let $\delta>0, u_{0} \in H^{2}$ and $u \in C\left(\left[0, T^{*}\right), H^{2}\right)$ a solution to (1.1). Then, there exists $T_{\delta}>0$ such that

$$
\|u\|_{L_{T}^{6}\left(W^{2,3}\right)} \leq \delta, \quad \text { for all } \quad 0 \leq T \leq T_{\delta} .
$$

Proof. Let $v:=e^{i t \Delta^{2}} u_{0}$ to be the solution to the free associated problem and $w=u-v$. By the Strichartz estimate (1.6), we have

$$
\|w\|_{L_{T}^{6}\left(W^{2,3}\right)} \lesssim\|g(u)\|_{L_{T}^{\frac{6}{5}}\left(W^{2, \frac{3}{2}}\right)} \cdot
$$

We have

$$
\begin{aligned}
\|\Delta(g(u))\|_{L_{T}^{\frac{6}{5}}\left(L^{\frac{3}{2}}\right)} & \leq\left\|\Delta u g^{\prime}(u)\right\|_{L_{T}^{\frac{6}{5}}\left(L^{\frac{3}{2}}\right)}+\left\||\nabla u|^{2} g^{\prime \prime}(u)\right\|_{L_{T}^{\frac{6}{5}}\left(L^{\frac{3}{2}}\right)} \\
& \leq(I)+(I I) .
\end{aligned}
$$

Using a continuity argument, there exists $T>0$ such that $\|u\|_{L_{T}^{\infty}\left(H^{2}\right)} \leq 1+\left\|u_{0}\right\|_{H^{2}}$. For $\varepsilon_{0}:=\frac{\pi^{2}}{\left(1+\left\|u_{0}\right\|_{H^{2}}\right)^{2}}$, by Moser-Trudinger inequality (1.8),

$$
\begin{aligned}
(I) & \lesssim\|\Delta u\|_{L_{T}^{\infty}\left(L^{2}\right)}\left\|e^{\varepsilon_{0}|u|^{2}}-1\right\|_{L_{T}^{\infty}\left(L^{6}\right)} T^{\frac{5}{6}} \\
& \lesssim\|\Delta u\|_{L_{T}^{\infty}\left(L^{2}\right)}\left\|e^{6 \varepsilon_{0}\|u-\Delta u\|^{2}\left(\frac{|u|}{\|u-\Delta u\|}\right)^{2}}-1\right\|_{L_{T}^{\infty}\left(L^{1}\right)}^{\frac{1}{6}} T^{\frac{5}{6}} \\
& \lesssim \mathcal{K}^{\frac{1}{6}}\|u\|_{L_{T}^{\infty} H^{2}} T^{\frac{5}{6}} \rightarrow 0 \quad \text { as } \quad T \rightarrow 0
\end{aligned}
$$


Let estimate $(I I)=\left\||\nabla u|^{2} g^{\prime \prime}(u)\right\|_{L_{T}^{\frac{6}{5}\left(L^{\frac{3}{2}}\right)}}$. Using the Sobolev embedding

$$
\left.\left.H^{1}\left(\mathbb{R}^{4}\right) \hookrightarrow L^{q}\left(\mathbb{R}^{4}\right) \quad \text { for all } \quad q \in\right] 1,4\right],
$$

with Hölder inequality, we have

$$
\begin{aligned}
(I I) & \lesssim\left\||\nabla u|^{2}\left(e^{\frac{\varepsilon_{0}}{6}|u|^{2}}-1\right)\right\|_{L_{T}^{\frac{6}{5}}\left(L^{\frac{3}{2}}\right)} \\
& \lesssim\|\nabla u\|_{L_{T}^{\infty}\left(L^{4}\right)}^{2}\left\|e^{\frac{\varepsilon_{0}}{6}|u|^{2}}-1\right\|_{L_{T}^{\frac{6}{5}}\left(L^{6}\right)} \\
& \lesssim\|u\|_{L_{T}^{\infty}\left(H^{2}\right)}^{2}\left(\left\|e^{\varepsilon_{0}|u|^{2}}-1\right\|_{L_{T}^{\infty}\left(L^{1}\right)}\right)^{\frac{1}{6}} T^{\frac{5}{6}} \\
& \lesssim \mathcal{K}^{\frac{1}{6}}\|u\|_{L_{T}^{\infty}\left(H^{2}\right)}^{2} T^{\frac{5}{6}} \rightarrow 0, \quad \text { as } \quad T \rightarrow 0 .
\end{aligned}
$$

Arguing as previously we have

$$
\begin{aligned}
\|g(u)\|_{L_{T}^{\frac{6}{5}}\left(L^{\frac{3}{2}}\right)} & \lesssim\left\|e^{\frac{5}{6} \varepsilon_{0}|u|^{2}}-1\right\|_{L_{T}^{\frac{6}{5}}\left(L^{\frac{3}{2}}\right)} \\
& \lesssim\left\|e^{\varepsilon_{0}|u|^{2}}-1\right\|_{L_{T}^{\frac{5}{6}}\left(L^{1}\right)} T^{\frac{5}{6}} \\
& \lesssim \mathcal{K}^{\frac{5}{6}} T^{\frac{5}{6}} \rightarrow 0 \text { as } T \rightarrow 0 .
\end{aligned}
$$

Which implies that

$$
\|w\|_{L_{T}^{6}\left(W^{2,3}\right)} \leq C_{T} \rightarrow 0, \quad \text { as } \quad T \rightarrow 0 .
$$

Thus, by the energy conservation of the free solution $v$,

$$
\|u\|_{L_{T}^{6}\left(W^{2,3}\right)} \leq C T\left\|u_{0}\right\|_{H^{2}}+C_{T} \rightarrow 0 \quad \text { as } \quad T \rightarrow 0
$$

The proof is closed.

3.3. Global existence in the defocusing case. This subsection is devoted to prove that the solution given by Theorem 2.1 is global in the case $\mu=1$. We recall an important fact that is the time of local existence depends only on the quantity $\left\|u_{0}\right\|_{H^{2}\left(\mathbb{R}^{4}\right)}$. Let $u$ to be the unique maximal solution of (1.1) in the space $\mathcal{E}_{T}$ for any $T<T^{*}$, where $0<T^{*} \leq+\infty$ is the lifespan of $u$. We shall prove that $u$ is global. By contradiction, suppose that $T^{*}<+\infty$, we consider for $0<s<T^{*}$, the following problem

$$
\left(\mathcal{P}_{s}\right)\left\{\begin{array}{ccc}
i \partial_{t} v+\Delta^{2} v+v G^{\prime}\left(|v|^{2}\right) & = & 0 \\
v(s, .) & = & u(s, .) .
\end{array}\right.
$$

By the same arguments used in the local existence, and taking

$$
0<\varepsilon \leq \frac{\pi^{2}}{(1+2 E(u(0)))},
$$

instead of $\varepsilon_{0}$, we can find a real $\tau>0$ and a solution $v$ to $\left(\mathcal{P}_{s}\right)$ on $[s, s+\tau]$. According to the section of local existence, and using the conservation of energy, $\tau$ does not depend on $s$. Thus, if we let $s$ be close to $T^{*}$ such that $s+\tau>T^{*}$, we can extend $v$ for times higher than $T^{*}$. This fact contradicts the maximality of $T^{*}$. We obtain the result claimed in Theorem 2.1 . 


\section{The STATIONARY PROBlem}

The goal of this section to prove that the elliptic problem associated to (1.1) has a ground state in the meaning that it has a nontrivial positive radial solution which minimizes of the action $S$ when $K_{\alpha, \beta}$ vanishes.

\section{Remark 4.1.}

(1) The proof of Theorem 2.2 is based on several Lemmas.

(2) In this section, we write, for easy notation, $\phi^{\lambda}=\phi_{\alpha, \beta}^{\lambda}, K=K_{\alpha, \beta}, K^{Q}=$ $K_{\alpha, \beta}^{Q}, K^{N}=K_{\alpha, \beta}^{N}, \mathcal{L}=\mathcal{L}_{\alpha, \beta}, H=H_{\alpha, \beta}$ and $m=m_{\alpha, \beta}$.

Lemma 4.2. Let $(0,0) \neq(\alpha, \beta) \in \mathbb{R}_{+}^{2}$. Then

(1) $\min (\mathcal{L} H(\phi), H(\phi)) \geq 0$, for all $\phi \in H^{2}$.

(2) If $\alpha \neq 0$ then $\min (\mathcal{L} H(\phi), H(\phi))>0$, for all $0 \neq \phi \in H^{2}$.

(3) $\lambda \mapsto H\left(\phi^{\lambda}\right)$ is increasing.

Proof. With (1.5) yields $H(\phi)=\frac{1}{\alpha+2 \beta}\left[2 \beta\|\Delta \phi\|^{2}+\alpha \int_{\mathbb{R}^{4}}(D-1) G\left(|\phi|^{2}\right) d x\right] \geq 0$. Moreover, with a computation

$$
\begin{aligned}
\mathcal{L} H(\phi) & =\mathcal{L}\left(1-\frac{\mathcal{L}}{\alpha+2 \beta}\right) S(\phi) \\
& =-\frac{1}{\alpha+2 \beta}(\mathcal{L}-\alpha)(\mathcal{L}-(\alpha+2 \beta)) S(\phi)+\alpha\left(1-\frac{\mathcal{L}}{\alpha+2 \beta}\right) S(\phi) \\
& =-\frac{1}{\alpha+2 \beta}(\mathcal{L}-\alpha)(\mathcal{L}-(\alpha+2 \beta)) S(\phi)+\alpha H(\phi) .
\end{aligned}
$$

Now, since $(\mathcal{L}-\alpha)\|\Delta \phi\|^{2}=(\mathcal{L}-(\alpha+2 \beta))\|\phi\|^{2}=0$, we have $(\mathcal{L}-\alpha)(\mathcal{L}-(\alpha+$ $2 \beta))\|\phi\|_{H^{2}}^{2}=0$, and $\mathcal{L} G\left(|\phi|^{2}\right)=(\alpha D+2 \beta) G\left(|\phi|^{2}\right)$. So

$$
\begin{aligned}
\mathcal{L} H(\phi) & \geq \frac{1}{\alpha+2 \beta}(\mathcal{L}-\alpha)(\mathcal{L}-(\alpha+2 \beta)) \int_{\mathbb{R}^{4}} G\left(|\phi|^{2}\right) d x \\
& =\frac{\alpha}{\alpha+2 \beta} \int_{\mathbb{R}^{4}}\left(\alpha(D-1)^{2}+2 \beta(D-1)\right) G\left(|\phi|^{2}\right) d x \\
& \geq 0 .
\end{aligned}
$$

The last inequality is by (1.5). The two first points of the Lemma follow. The last point is a consequence of the equality $\partial_{\lambda} H\left(\phi^{\lambda}\right)=\mathcal{L} H\left(\phi^{\lambda}\right)$.

The next intermediate result is the following

Lemma 4.3. Let $\alpha>0, \beta \in \mathbb{R}$ and $\left(\phi_{n}\right)$ a bounded sequence of $H^{2}-\{0\}$ such that $\lim _{n} K^{Q}\left(\phi_{n}\right)=0$. Then, there exists $n_{0} \in \mathbb{N}$ such that $K\left(\phi_{n}\right)>0$ for all $n \geq n_{0}$.

Proof. We have, for some $p>2,|r g(r)|+\left|G\left(r^{2}\right)\right| \lesssim r^{p}\left(e^{r^{2}}-1\right)$. In fact, by the first equation of (1.5), the ratio tends to zero on infinity and using (1.3), the ratio 
is bounded near zero. Thus, for any $q \geq 1$,

$$
\begin{aligned}
K^{N}(v) & \lesssim \int_{\mathbb{R}^{4}}|v|^{p}\left(e^{|v|^{2}}-1\right) d x \\
& \lesssim\|v\|_{q p}^{p}\left\|e^{|v|^{2}}-1\right\|_{q^{\prime}} \\
& \lesssim\|v\|_{q p}^{p}\left\|e^{q^{\prime}|v|^{2}}-1\right\|_{1}^{\frac{1}{q^{\prime}}} .
\end{aligned}
$$

Now, if $q^{\prime 2}\|v-\Delta v\|^{2}<32 \pi^{2}$, by Moser-Trudinger inequality $K^{N}(v) \lesssim\|v\|_{q p}^{p}$. By the interpolation inequality

$$
\|v\|_{L^{r}\left(\mathbb{R}^{4}\right)} \lesssim\|v\|_{L^{2}\left(\mathbb{R}^{4}\right)}^{\frac{2}{r}}\|\Delta v\|_{L^{2}\left(\mathbb{R}^{4}\right)}^{1-\frac{2}{r}}, \quad \forall r \in[2, \infty),
$$

we have $K^{N}(v) \lesssim\|v\|_{q p}^{p} \lesssim\|v\|^{\frac{2}{q}}\|\Delta v\|^{p-\frac{2}{q}}$. Since $\left\|\Delta \phi_{n}\right\|^{2} \lesssim K^{Q}\left(\phi_{n}\right)$, the proof is achieved via taking $q$ such that $p-\frac{2}{q}>2$.

The last Lemma of this section reads

Lemma 4.4. Let $\alpha>0$ and $\beta \geq 0$. Then

$$
m_{\alpha, \beta}=\inf _{0 \neq \phi \in H_{r d}^{2}}\left\{H_{\alpha, \beta}(\phi), \text { s.t } K_{\alpha, \beta}(\phi) \leq 0\right\} .
$$

Proof. Let $m_{1}$ the right hand side, then it is sufficient to prove that $m \leq m_{1}$. Take $\phi \in H^{2}$ such that $K_{\alpha, \beta}(\phi)<0$ then by Lemma 4.3, the fact that $\lim _{\lambda \rightarrow-\infty} K_{\alpha, \beta}^{Q}\left(\phi^{\lambda}\right)=$ 0 and $\lambda \mapsto H\left(\phi_{\alpha, \beta}^{\lambda}\right)$ is increasing, there exists $\lambda<0$ such that

$$
K_{\alpha, \beta}\left(\phi^{\lambda}\right)=0, H_{\alpha, \beta}\left(\phi^{\lambda}\right) \leq H(\phi) .
$$

The proof is closed.

\section{Proof of Theorem 2.2}

First case $\alpha \neq 0$.

Let $\left(\phi_{n}\right)$ a minimizing sequence, namely

$$
0 \neq \phi_{n} \in H_{r d}^{2}, K\left(\phi_{n}\right)=0 \text { and } \lim _{n} H\left(\phi_{n}\right)=\lim _{n} S\left(\phi_{n}\right)=m .
$$

- First step: $\left(\phi_{n}\right)$ is bounded in $H^{2}$.

We have $\alpha\left[\left\|\phi_{n}\right\|_{H^{2}}^{2}-\int_{\mathbb{R}^{4}}\left|\phi_{n}\right| g\left(\left|\phi_{n}\right|\right) d x\right]=2 \beta\left[\int_{\mathbb{R}^{4}} G\left(\left|\phi_{n}\right|^{2}\right) d x-\left\|\phi_{n}\right\|^{2}\right]$ and $\left(\left\|\phi_{n}\right\|_{H^{2}}^{2}-\int_{\mathbb{R}^{4}} G\left(\left|\phi_{n}\right|^{2}\right) d x\right) \rightarrow m$. Denoting $\lambda:=\frac{2 \beta}{\alpha}$, yields

$$
\left\|\phi_{n}\right\|_{H^{2}}^{2}-\int_{\mathbb{R}^{4}}\left|\phi_{n}\right| g\left(\left|\phi_{n}\right|\right) d x=\lambda\left[\left\|\Delta \phi_{n}\right\|^{2}-\left\|\phi_{n}\right\|_{H^{2}}^{2}+\int_{\mathbb{R}^{4}} G\left(\left|\phi_{n}\right|^{2}\right) d x\right] .
$$

So, the following sequences are bounded

$$
\left(-\lambda\left\|\Delta \phi_{n}\right\|^{2}+\left\|\phi_{n}\right\|_{H^{2}}^{2}-\int_{\mathbb{R}^{4}}\left|\phi_{n}\right| g\left(\left|\phi_{n}\right|\right) d x\right), \quad\left(\left\|\phi_{n}\right\|_{H^{2}}^{2}-\int_{\mathbb{R}^{4}} G\left(\left|\phi_{n}\right|^{2}\right) d x\right) .
$$

Thus, for any real number $a$, the following sequence is also bounded

$$
\lambda\left\|\Delta \phi_{n}\right\|^{2}+(a-1)\left\|\phi_{n}\right\|_{H^{2}}^{2}+\int_{\mathbb{R}^{4}}(D-a) G\left(\left|\phi_{n}\right|^{2}\right) d x .
$$


Now, taking $a>1$ near to one and using the assumption (1.5), yields that $\left(\phi_{n}\right)$ is bounded in $H^{2}$.

- Second step: the limit of $\phi_{n}$ is nonzero and $m>0$.

Taking account of the compact injection of the radial Sobolev space $H_{r d}^{2}\left(\mathbb{R}^{4}\right)$ on the Lebesgue space $L^{p}\left(\mathbb{R}^{4}\right)$ for any $2<p<\infty$, we take

$$
\phi_{n} \rightarrow \phi \text { in } H^{2} \text { and } \phi_{n} \rightarrow \phi \text { in } L^{p}, \forall p \in(2, \infty) .
$$

By (1.5), for some $p>2$ and $a>0$ small enough

$$
\begin{aligned}
\max \left\{\int_{\mathbb{R}^{4}} G\left(\left|\phi_{n}\right|^{2}\right) d x, \int_{\mathbb{R}^{4}}\left|\phi_{n}\right| g\left(\left|\phi_{n}\right|\right) d x\right\} & \lesssim\left\|\phi_{n}^{p}\left(e^{a\left|\phi_{n}\right|^{2}}-1\right)\right\|_{1} \\
& \lesssim\left\|\phi_{n}\right\|_{2 p}^{p}\left\|e^{2 a\left|\phi_{n}\right|^{2}}-1\right\|_{1}^{\frac{1}{2}} \\
& \lesssim\left\|\phi_{n}\right\|_{2 p}^{p}\left\|\phi_{n}\right\| \rightarrow 0 .
\end{aligned}
$$

In the last lines, we used the fact that $\left(\phi_{n}\right)$ is bounded in $H^{2}$ via Moser-Trudinger inequality. If we assume that $\phi=0$, by Lemma 4.3, $K\left(\phi_{n}\right)>0$ for large $n$ which is absurd. So

$$
\phi \neq 0
$$

By the Moser-Trudinger inequality via (1.3) with (1.5), the mean value theorem and the convexity of the exponential, for any $\varepsilon>0$,

$$
\begin{aligned}
\int_{\mathbb{R}^{4}}\left|G\left(|\phi|^{2}\right)-G\left(\left|\phi_{n}\right|^{2}\right)\right| d x & \lesssim \int_{\mathbb{R}^{4}}\left|\phi-\phi_{n}\right|\left(\left|\phi_{n}\right|+|\phi|\right)\left(e^{\varepsilon\left|\phi_{n}\right|^{2}}-1+e^{\varepsilon|\phi|^{2}}-1\right) d x \\
& \lesssim\left\|\phi-\phi_{n}\right\|_{3}\left(\left\|\phi_{n}\right\|_{3}+\|\phi\|_{3}\right)\left(\left\|\phi_{n}\right\|^{\frac{2}{3}}+\|\phi\|^{\frac{2}{3}}\right) .
\end{aligned}
$$

Thus

$$
\int_{\mathbb{R}^{4}} G\left(\left|\phi_{n}\right|^{2}\right) d x \longrightarrow \int_{\mathbb{R}^{4}} G\left(|\phi|^{2}\right) d x .
$$

Similarly, we have

$$
\int_{\mathbb{R}^{4}}\left|\phi_{n}\right| g\left(\left|\phi_{n}\right|\right) d x \longrightarrow \int_{\mathbb{R}^{4}}|\phi| g(|\phi|) d x .
$$

So, with lower semi continuity of $H^{2}$ norm, we have

$$
\begin{aligned}
0 & =\liminf _{n} K\left(\phi_{n}\right) \\
& \geq \alpha \liminf _{n}\left\|\Delta \phi_{n}\right\|^{2}+(\alpha+2 \beta) \liminf _{n}\left\|\phi_{n}\right\|^{2}-\int_{\mathbb{R}^{4}}(\alpha D+2 \beta) G\left(|\phi|^{2}\right) d x \\
& \geq K(\phi) .
\end{aligned}
$$

Similarly, we have $H(\phi) \leq m$. Using (4.3), we can assume that $K(\phi)=0$ and $S(\phi)=H(\phi) \leq m$. So that $\phi$ is a minimizer satisfying (4.4). Since $H(\phi)=$ $\frac{1}{\alpha+2 \beta}\left[2 \beta\|\Delta \phi\|^{2}+\alpha \int_{\mathbb{R}^{4}}(D-1) G\left(|\phi|^{2}\right) d x\right]$, via the assumption (1.5), we have

$$
m>0 \text {. }
$$


- Third step: $\phi$ is a solution to (2.1).

Now, there is a Lagrange multiplier $\eta \in \mathbb{R}$ such that $S^{\prime}(\phi)=\eta K^{\prime}(\phi)$. Thus

$$
\begin{aligned}
0=K(\phi) & =\mathcal{L} S(\phi)=\left\langle S^{\prime}(\phi), \mathcal{L}(\phi)\right\rangle \\
& =\eta\left\langle K^{\prime}(\phi), \mathcal{L}(\phi)\right\rangle \\
& =\eta \mathcal{L} K(\phi)=\eta \mathcal{L}^{2} S(\phi)
\end{aligned}
$$

With a previous computation and taking account of the second equation of (1.5),

$$
\begin{aligned}
-\mathcal{L}^{2} S(\phi)-\alpha(\alpha+2 \beta) S(\phi) & =-(\mathcal{L}-(\alpha+2 \beta))(\mathcal{L}-\alpha) S(\phi) \\
& =\alpha \int_{\mathbb{R}^{4}}\left(\alpha(D-1)^{2}+2 \beta(D-1)\right) G\left(|\phi|^{2}\right) d x \\
& \geq 0 .
\end{aligned}
$$

Thus $\eta=0$ and $S^{\prime}(\phi)=0$. So, $\phi$ is a ground state and $m$ is independent of $\alpha, \beta$. Second case $\alpha=0$.

Without loss of generality, we take $\beta=1$. Let $\left(\phi_{n}\right)$ a minimizing sequence. Since (4.4) is invariant by the scaling $\phi_{n}\left(\frac{\cdot}{\sqrt{\left\|\phi_{n}\right\|}}\right)$ because $\left\|\Delta \phi_{n}\left(\frac{\cdot}{\sqrt{\left\|\phi_{n}\right\|}}\right)\right\|^{2}=\left\|\Delta \phi_{n}\right\|^{2}$ and $K_{0,1}\left(\phi_{n}\left(\frac{\cdot}{\sqrt{\left\|\phi_{n}\right\|}}\right)\right)=0$. Thus, we may assume that $\left\|\phi_{n}\right\|=1$. Moreover, $H\left(\phi_{n}\right)=\left\|\Delta \phi_{n}\right\|^{2} \rightarrow m$, thus $\phi_{n}$ is bounded in $H^{2}$. Using compact Sobolev embedding as previously, we take $\phi_{n} \rightarrow \phi$ in $H^{2}$ and $\phi_{n} \rightarrow \phi$ in $L^{p}, \forall p \in(2, \infty)$. Now, by the fact that

$$
0=K\left(\phi_{n}\right)=1-\int_{\mathbb{R}^{4}} G\left(\left|\phi_{n}\right|^{2}\right) d x \rightarrow 1-\int_{\mathbb{R}^{4}} G\left(|\phi|^{2}\right) d x,
$$

we have

$$
\phi \neq 0 \text {. }
$$

Using (1.3), for $\lambda=0^{+},\left|K^{N}(\lambda \phi)\right|=o\left(K^{Q}(\lambda \phi)\right)=\lambda^{2} K^{Q}(\phi)$. Thus

$$
K(\phi)<0 \Rightarrow \exists \lambda \in(0,1), / K(\lambda \phi)=0, H(\lambda \phi) \leq H(\phi)=\|\Delta \phi\|^{2} .
$$

So, we may assume that $K(\phi)=0$ and $S(\phi)=H(\phi) \leq m$. Thus $m=H(\phi)=$ $\|\Delta \phi\|^{2}>0$. Now, with a Lagrange multiplicator $\eta$, we have $S^{\prime}(\phi)=\frac{\eta}{2} K^{\prime}(\phi)$. Then $\Delta^{2} \phi=(\eta-1)(\phi-g(\phi))$. Moreover, since $\langle\phi, \phi-g(\phi)\rangle_{L^{2}}=\frac{1}{2} K_{0,1}(\phi)-$ $\int_{\mathbb{R}^{4}}(D-1) G\left(|\phi|^{2}\right)<0$, then $(\eta-1)<0$. Taking a positive real $\lambda$, we have

$$
\Delta^{2}\left(\phi\left(e^{-\lambda} .\right)\right)=e^{-4 \lambda}(\eta-1)\left[\phi\left(e^{-\lambda} .\right)-g\left(\phi\left(e^{-\lambda} .\right)\right)\right] .
$$

Finally, for $\lambda$ such that $e^{-4 \lambda}(\eta-1)=-1$, we have a ground state, which concludes the proof.

\section{Global Well-Posedness in the focusing CASE}

In this section, we prove Theorem 2.3 about global well-psedness of the focusing case associated to (1.1). Let us start with an auxiliary result.

Lemma 5.1. The set $A_{\alpha, \beta}^{+}$is invariant under the flow of (1.1). 
Proof. Let $u_{0} \in A_{\alpha, \beta}^{+}$and $u \in C_{T^{*}}\left(H^{2}\right)$ the maximal solution to (1.1). Assume that there exists some time $t_{0} \in\left(0, T^{*}\right)$ such that $u\left(t_{0}\right) \notin A_{\alpha, \beta}^{+}$. Since $S(u)$ is conserved, we have $K_{\alpha, \beta}\left(u\left(t_{0}\right)\right) \leq 0$. So, with a continuity argument, there exists a positive time $t_{1} \in\left(0, t_{0}\right)$ such that $K_{\alpha, \beta}\left(u\left(t_{1}\right)\right)=0$. This contradicts the definition of $m_{\alpha, \beta}$. The proof is finished.

Lemma 5.2. Let $(0,0) \neq(\alpha, \beta) \in \mathbb{R}_{+}^{2}$. Then, the set $A_{\alpha, \beta}^{+}$is independent of $(\alpha, \beta)$.

Proof. Let $(\alpha, \beta)$ and $\left(\alpha^{\prime}, \beta^{\prime}\right)$ in $\mathbb{R}_{+}^{2}-\{(0,0)\}$. We denote, for $\delta \geq 0$, the sets

$$
\begin{array}{llll}
A_{\alpha, \beta}^{+\delta}:=\left\{v \in H^{2}\right. & \text { s. t } \quad S(v)<m-\delta & \text { and } & \left.K_{\alpha, \beta}(v) \geq 0\right\} ; \\
A_{\alpha, \beta}^{-\delta}:=\left\{v \in H^{2}\right. & \text { s. t } \quad S(v)<m-\delta & \text { and } & \left.K_{\alpha, \beta}(v)<0\right\} .
\end{array}
$$

By Theorem 2.2, the reunion $A_{\alpha, \beta}^{+\delta} \cup A_{\alpha, \beta}^{-\delta}$ is independent of $(\alpha, \beta)$. So, it is sufficient to prove that $A_{\alpha, \beta}^{+\delta}$ is independent of $(\alpha, \beta)$. If $S(\phi)<m$ and $K_{\alpha, \beta}(\phi)=0$, then $\phi=0$. So $A_{\alpha, \beta}^{+\delta}$ is open. The rescaling $\phi^{\lambda}:=e^{\alpha \lambda} \phi\left(e^{-\beta \lambda}\right.$. ) implies that a neighborhood of zero is in $A_{\alpha, \beta}^{+\delta}$. Moreover, this rescaling with $\lambda \rightarrow-\infty$ gives that $A_{\alpha, \beta}^{+\delta}$ is contracted to zero and so it is connected. Now, by the definition, $A_{\alpha, \beta}^{-\delta}$ is open, and $0 \in A_{\alpha, \beta}^{+\delta} \cap A_{\alpha^{\prime}, \beta^{\prime}}^{+\delta}$. Write $A_{\alpha, \beta}^{+\delta}=\left(A_{\alpha, \beta}^{+\delta} \cap A_{\alpha^{\prime}, \beta^{\prime}}^{+\delta}\right) \cup\left(A_{\alpha, \beta}^{+\delta} \cap A_{\alpha^{\prime}, \beta^{\prime}}^{-\delta}\right)$. Using a connexity argument, we have $A_{\alpha, \beta}^{+\delta}=A_{\alpha^{\prime}, \beta^{\prime}}^{+\delta}$. The proof is achieved.

Now, we prove Theorem 2.3. With a translation argument, we assume that $u_{0} \in A_{\alpha, \beta}^{+}$, for some positive real numbers $(0,0) \neq(\alpha, \beta) \in \mathbb{R}_{+}^{2}$. By Lemmas 5.1, $u(t) \in A_{\alpha, \beta}^{+}$for any $t \in\left[0, T^{*}\right)$. Using Lemma $5.2, u(t) \in A_{1,1}^{+}$for any $t \in\left[0, T^{*}\right)$. Thus,

$$
\begin{aligned}
m & \geq\left(S-K_{1,1}\right)(u) \\
& =H_{1,1}(u) \\
& =\frac{1}{3}\left[2\|\Delta u\|^{2}+\int_{\mathbb{R}^{4}}(D-1) G\left(|u|^{2}\right) d x\right] \\
& \geq \frac{2}{3}\|\Delta u\|^{2} .
\end{aligned}
$$

Moreover, since the $L^{2}$ norm of $u$ is conserved, we have

$$
\sup _{0 \leq t \leq T^{*}}\|u(t)\|_{H^{2}}<\infty .
$$

Thus $T^{*}=\infty$. This ends the proof.

\section{REFERENCES}

1. S. Adachi and K. Tanaka, Trudinger type inequalities in $\mathbb{R}^{N}$ and their best exponent, Proc. Amer. Math. Soc. 128 (1999), no. 7, 2051-2057.

2. H. Bahouri, S. Ibrahim and G. Perleman, Scattering for the critical 2-D NLS with exponential growth, arXiv:1302.1269 [math.AP].

3. J. Bourgain, Global wellposedness of defocusing critical nonlinear Schrödinger equation in the radial case, J. Amer. Math. Soc. 12 (1999), 145-171.

4. T. Cazenave and F.B. Weissler, The Cauchy problem for the critical nonlinear Schrödinger equation in $H^{s}$, Nonl. Anal. - TMA. 14 (1990), 807-836. 
5. J. Colliander, S. Ibrahim, M. Majdoub and N. Masmoudi, Energy critical NLS in two space dimensions, J. Hyperbolic Differ. Equ, 6 (2009), no. 3, 549-575.

6. J. Colliander, M. Keel, G. Staffilani, H. Takaoka, and T. Tao, Global well-posedness and scattering for the energy-critical nonlinear Schrödinger equation in $R^{3}$, Ann. Math. 167 (2008), 767-865.

7. S. Ibrahim, M. Majdoub, N. Masmoudi and K. Nakanishi, Scattering for the $2 D$ energy critical wave equation, Duke Math. 150 (2009), no. 2, 287-329.

8. V.I. Karpman, Stabilization of soliton instabilities by higher-order dispersion: fourth-order nonlinear Schrödinger equations, Phys. Rev. E 53 (1996), no. 2, 1336-1339.

9. V.I. Karpman and A.G. Shagalov, Stability of soliton described by nonlinear Schrödinger type equations with higher-order dispersion, Phys D. 144 (2000), 194-210.

10. C.E. Kenig and F. Merle, Global well-posedness, scattering and blow up for the energycritical, focusing, nonlinear Schrödinger equation in the radial case, Invent. Math. 166 (2006), 645-675.

11. O. Mahouachi and T. Saanouni, Global well posedness and linearization of a semilinear wave equation with exponential growth, Georgian Math. J. 17 (2010), 543-562.

12. O. Mahouachi and T. Saanouni, Well and ill posedness issues for a $2 D$ wave equation with exponential nonlinearity, J. Partial Differ. Equ. 24 (2011), no. 4, 361-384.

13. C. Miao, G. Xu and L. Zhao, Global well-posedness and scattering for the focusing energy-critical nonlinear Schrödinger equations of fourth-order in the radial case, Arxiv: 0807.06090v2 [math.AP], (2008).

14. C. Miao, G. Xu and L. Zhao, Global well-posedness and scattering for the defocusing energy-critical nonlinear Schrödinger equations of fourth-order in dimensions $d \geq 9$, Arxiv: 0807.06092v2 [math.AP], (2009).

15. M. Nakamura and T. Ozawa, Nonlinear Schrödinger equations in the Sobolev space of critical order, J. Funct. Anal. 155 (1998), 364-380.

16. J. Moser, A sharp form of an inequality of N. Trudinger, Ind. Univ. Math. J. 20 (1971), 1077-1092.

17. B. Pausader, Global well-posedness for energy critical fourth-order Schrödinger equations in the radial case, Dyn. Partial Differ. Equ. 4 (2007), no. 3, 197-225.

18. B. Pausader, The cubic fourth-order Schrödinger equation, J. Funct. Anal. 256 (2009), 2473-2517.

19. L.E. Payne and D.H. Sattinger, Saddle Points and Instability of Nonlinear Hyperbolic Equations, Israel J. Math. 22 (1975), no. 3-4, 273-303.

20. L. Peletier and W.C. Troy, Spatial patterns, Higher order models in physics and mechanics, Progress in Nonlinear Differential Equations and their Applications, 45. Birkhuser Boston, Inc., Boston, MA, 2001.

21. B. Ruf, A sharp Moser-Trudinger type inequality for unbounded domains in $\mathbb{R}^{2}$, J. Funct. Anal. 219 (2004), 340-367.

22. B. Ruf and S. Sani, sharp Adams-type inequalities in $\mathbb{R}^{n}$, Trans. Amer. Math. Soc. 365 (2013), 645-670.

23. E. Ryckman and M. Visan, Global well-posedness and scattering for the defocusing energycritical nonlinear Schrödinger equation in $R^{1+4}$, Amer. J. Math. 129 (2007), 1-60.

24. T. Saanouni, Global well-posedness and scattering of a $2 D$ Schrödinger equation with exponential growth, Bull. Belg. Math. Soc. 17 (2010), 441-462.

25. T. Saanouni, Decay of Solutions to a $2 D$ Schrödinger Equation, J. Partial Differ. Equ. 24 (2011), no. 1, 37-54.

26. T. Saanouni, Remarks on the semilinear Schrödinger equation, J. Math. Anal. Appl. 400 (2013), 331-344.

27. T. Saanouni, Scattering of a $2 D$ Schrödinger equation with exponential growth in the conformal space, Math. Meth. Appl. Sci. 33 (2010), 1046-1058. 
28. T. Saanouni, Global well-posedness and instability of a $2 D$ Schrödinger equation with harmonic potential in the conformal space, J. Abstr. Differ. Equ. Appl. 4 (2013), no. 1, $23-42$.

29. T. Saanouni, Blowing-up semilinear wave equation with exponential nonlinearity in two space dimensions, Proc. Indian Acad. Sci. (Math. Sci.) 123, no. 3 (2013), 365-372.

30. T. Saanouni, Global well-posedness of a damped Schrödinger equation in two space dimensions, Math. Methods Appl. Sci. 37 (2014), no. 4, 488-495.

31. T. Tao, Global well-posedness and scattering for the higher-dimensional energycritical nonlinear Schrödinger equation for radial data, New York J. Math. 11 (2005), 57-80.

32. N.S. Trudinger, On imbedding into Orlicz spaces and some applications, J. Math. Mech. 17 (1967), 473-484.

33. M. Visan, The defocusing energy-critical nolinear Schrödinger equation in higher dimensions, Duke. Math. J. 138 (2007), 281-374.

University of Tunis El Manar, Faculty of Science of Tunis, LR03ES04 Partial DifFERENTIAL EQUATIONS AND APPLiCATIONS, 2092 Tunis, Tunisia.

E-mail address: Tarek.saanouni@ipeiem.rnu.tn 